\title{
Venous thromboembolism prophylaxis in mental healthcare: do the benefits outweigh the risks?
}

\author{
Rashmi Patel ${ }^{1}$ \\ ${ }^{1}$ King's College London, UK \\ Correspondence to Rashmi Patel \\ (rcpsych@rpatel.co.uk) \\ First received 28 Dec 2013, final \\ revision 18 May 2014, accepted \\ 10 Jun 2014 \\ (C) 2014 The Author. This is an open- \\ access article published by the Royal \\ College of Psychiatrists and distributed \\ under the terms of the Creative \\ Commons Attribution License (http:// \\ creativecommons.org/licenses/by/ \\ 3.0), which permits unrestricted use, \\ distribution, and reproduction in any \\ medium, provided the original work \\ is properly cited.
}

BJPsych Bulletin (2015), 39, 61-64, doi: 10.1192/pb.bp.113.046680

Summary Venous thromboembolism is an important cause of morbidity and mortality. In recent years, growing awareness has led to the development of strategies to prevent venous thromboembolism in individuals admitted to hospital who are deemed to be at high risk. However, there remains a considerable degree of uncertainty over whether these strategies are of overall benefit and there are few published studies on people who are admitted to psychiatric hospitals. In this editorial I review current clinical practice and areas of uncertainty with respect to venous thromboembolism prophylaxis and its implementation in mental healthcare settings.

Declaration of interest None.
Previous estimates have suggested that venous thromboembolism is responsible for around 60000 deaths in the UK each year. ${ }^{1}$ It is thought that individuals admitted to hospital may be at increased risk of developing deep vein thrombosis and/or pulmonary embolism as a result of reduced mobility or intercurrent illness. Other important risk factors include older age (over 60 years), active malignancy, dehydration, inherited or acquired thrombophilia, obesity, previous venous thromboembolism or family history of venous thromboembolism, oral contraceptive pill use, hormone replacement therapy, pregnancy and varicose veins with phlebitis. ${ }^{2,3}$ These risk factors may be applicable to individuals admitted to hospital for medical, surgical or psychiatric care. For these reasons, current clinical guidelines recommend a risk assessment for all people who are admitted to hospital and prescription of a low-dose anticoagulant such as low molecular weight heparin (LMWH) and/or mechanical prophylaxis for those thought to be at high risk. ${ }^{2}$ The decision as to whether to offer venous thromboembolism prophylaxis should be balanced against potential risks, particularly the risk of bleeding with LMWH.

\section{Evidence base for venous thromboembolism prophylaxis in acute general hospitals}

Numerous interventional studies have investigated the role of mechanical and pharmacological prophylaxis in reducing the risk of venous thromboembolism among those admitted to an acute general hospital. Studies have principally focused on patients undergoing orthopaedic surgery, nonorthopaedic surgery or no surgery. Interventional studies have demonstrated a reduction in symptomatic deep vein thrombosis among patients undergoing orthopaedic surgery who receive LMWH (relative risk $(\mathrm{RR})=0.50,95 \%$ CI $0.43-$ $0.59^{4}$ ). The use of LMWH in this group is not associated with a significant increase in major bleeding $(R R=0.81,95 \%$ CI $0.38-1.72^{4}$ ). Prophylactic LMWH is also associated with a reduction in non-fatal symptomatic venous thromboembolism in patients who are undergoing non-orthopaedic surgery $\left(R R=0.31,95 \%\right.$ CI $\left.0.12-0.81^{5}\right)$ and possibly a reduction in symptomatic deep vein thrombosis $(\mathrm{RR}=0.47$, 95\% CI $0.22-1.00^{6}$ ) and pulmonary embolism (odds ratio $(\mathrm{OR})=0.70,95 \%$ CI $0.56-0.87^{7}$ ) in patients who are not undergoing surgery. However, this is balanced with a significantly increased risk of major bleeding $(\mathrm{OR}=1.28$, $\left.1.05-1.56^{7}\right)$. Furthermore, when considering fatal pulmonary embolism or overall mortality, prophylactic LMWH is not associated with significant benefit in any group. ${ }^{4-7}$

\section{Evidence base for venous thromboembolism prophylaxis in mental healthcare settings}

In contrast to studies in acute general hospitals, there is relatively little published evidence investigating venous thromboembolism incidence and the role of pharmacological or mechanical prophylaxis in mental healthcare settings. A recent observational study that included systematic venous ultrasonography identified deep vein thrombosis in 10 out of 449 participants $(2.2 \%)$ following 10 days of admission to a psychiatric hospital. ${ }^{8}$ A total of 16 out of $458(3.5 \%)$ had experienced an episode of venous 
thromboembolism by 90 days following admission. Of these, three had a non-fatal pulmonary embolism. The study also showed that venous thromboembolism was more likely in older people (8.6\% of those aged over 75 years), which may relate to greater exposure to risk factors such as immobility. Another study based on a review of hospital records revealed 17 confirmed cases of venous thromboembolism among 1495 people (1.1\%) admitted to an in-patient mental health service for older people. ${ }^{9}$ This contrasts with an incidence of $2.4 \%$ within 91 days among people undergoing total hip arthroplasty surgery ${ }^{10}$ and 1.45 per 1000 person years in the general population. ${ }^{11}$

There is growing evidence from observational studies that suggests a possible association between antipsychotic medications and venous thromboembolism, particularly for clozapine and first-generation antipsychotics. ${ }^{12}$ However, it has been difficult to establish whether this association could be a direct pharmacological consequence of antipsychotics (leading to a prothrombotic state) or if it is mediated through other risk factors that are consequences of antipsychotics, such as obesity or sedation leading to reduced mobility. ${ }^{13}$ Some studies have also pointed towards physical restraint as a potential risk factor for venous thromboembolism in mental healthcare settings. ${ }^{14-16}$

\section{Areas of uncertainty}

A recent meta-analysis has led to increasing controversy over the potential benefits of pharmacological or mechanical measures to prevent venous thromboembolism among hospital patients who are not undergoing surgery. ${ }^{7}$ Although a reduction in non-fatal symptomatic venous thromboembolism was seen with LMWH prophylaxis, this is balanced with an increased risk of bleeding and no overall benefit in terms of reduced mortality. Furthermore, the relative benefit of prophylaxis only translates to a modest reduction in absolute risk; for every 1000 in-patients treated with LMWH, only three cases of pulmonary embolism are prevented balanced with four additional cases of major bleeding. ${ }^{7}$

There is also continued uncertainty about the true incidence of clinically significant venous thromboembolism. ${ }^{17}$ Although data from epidemiological modelling suggests that venous thromboembolism is responsible for around 60000 deaths each year in the UK, ${ }^{1}$ data from postmortem studies suggest a much lower rate of around 5680 per year. ${ }^{18}$ Whether pharmacological and mechanical prophylaxis could prevent all deaths from venous thromboembolism is also unclear.

\section{Do people who develop venous thromboembolism always need treatment with anticoagulants?}

Some observational studies have employed systematic ultrasound screening to identify asymptomatic as well as symptomatic deep vein thrombosis. Although deep vein thrombosis was identified in 10 out of 449 participants following admission to a psychiatric hospital, seven cases were of distal deep vein thrombosis of which only one case was symptomatic. ${ }^{8}$ The extent to which asymptomatic deep vein thrombosis predisposes an individual to increased risk of mortality remains uncertain, particularly with respect to asymptomatic distal deep vein thrombosis. ${ }^{19}$

The advent of computed tomography pulmonary angiography (CTPA) has led to a substantial increase in the radiological diagnosis of pulmonary embolism. ${ }^{20}$ However, uncertainty is growing over the optimum treatment particularly with respect to whether all those with a radiological diagnosis of pulmonary embolism would benefit from anticoagulation. ${ }^{21}$ It is thought that small subsegmental emboli may not necessarily be associated with adverse clinical outcomes and that the risks of bleeding from treatment with anticoagulants may outweigh any benefits within this group. ${ }^{22}$

\section{Benefits and risks of venous thromboembolism prophylaxis in mental healthcare settings}

There are no published interventional studies that have investigated the potential benefits of venous thromboembolism prophylaxis in mental healthcare in-patient settings. Despite this, there is ongoing interest in developing and utilising risk-screening tools to identify individuals at increased risk of venous thromboembolism for prophylaxis. ${ }^{23}$ Furthermore, there is no published evidence that has investigated the potential harms of venous thromboembolism prophylaxis in this setting. Although risks of bleeding have been well characterised for people admitted to acute general hospitals, it is not clear whether the same risks apply elsewhere. In particular, prolonged use of LMWH can predispose to thrombocytopenia leading to an increased risk of bleeding. ${ }^{24}$ The mean length of stay in an in-patient mental healthcare setting (adult: 52.1 days, older people: 93.2 days) is substantially greater than that of an acute medical unit (5.5 days). ${ }^{25}$ With the exception of those taking clozapine, full blood count monitoring is not routinely performed in the mental healthcare in-patient setting. The extent to which staff in mental healthcare settings are trained to administer prophylaxis and recognise potential adverse complications is also unclear. ${ }^{3}$ For these reasons, it is possible that the risk of thrombocytopenia from LMWH may be greater for those who receive it for venous thromboembolism prophylaxis in the mental healthcare setting.

Balancing the potential risks of bleeding and the potential benefits of preventing venous thromboembolism with pharmacological prophylaxis is problematic. Costutility analysis is a method by which the benefits and risks of an intervention may be balanced with respect to quality of life measures. A study investigating the application of cost-utility analysis to venous thromboembolism found that there was a wide degree of variation in individual estimates of cost-utility of both acute venous thromboembolism and bleeding complications from pharmacological prophylaxis. ${ }^{26}$ However, in the mental healthcare in-patient setting, it is sometimes not possible for patients to weigh up benefits and risks of an intervention because of lack of mental capacity. Furthermore, there is little evidence to estimate the potential benefits and risks of venous thromboembolism prophylaxis among individuals who lack 
capacity as randomised controlled trials have excluded these individuals. ${ }^{17}$

\section{Discussion}

Venous thromboembolism remains an important cause of mortality in people who are admitted to hospital. However, in recent years, there has been ongoing uncertainty over the efficacy and risks of prophylaxis among in-patients who are not undergoing surgery, ${ }^{6,7}$ and whether everyone with established venous thromboembolism would benefit from anticoagulant treatment. ${ }^{21,22}$ Although prophylaxis appears to reduce the incidence of non-fatal venous thromboembolism, there is no robust evidence that supports a reduction in mortality. ${ }^{4-7}$ This may be because of the balance with risk of bleeding for pharmacological prophylaxis., ${ }^{7,24}$ There is even less evidence to support its use in mental healthcare in-patient settings where no interventional studies have been published.

Despite this, substantial resources (over $£ 30$ million per year in England) have been invested into venous thromboembolism prevention programmes that claim to 'save lives'. ${ }^{27}$ Although it is claimed these investments have resulted in a modest overall saving (a yield of $2.7 \%^{28}$ ), it is possible that there is a greater opportunity cost in mental healthcare settings where there is currently no evidence for the cost-effectiveness of venous thromboembolism prophylaxis.

It is clear that there is an ongoing need to improve the overall physical health of individuals with mental illness, particularly those with severe mental illness who have been shown to have a substantially lower life expectancy than the general population. ${ }^{29}$ Although venous thromboembolism is an important cause of mortality, a greater degree of impact could be achieved by investing resources into improving detection and treatment of new cases $^{3}$ as well as preventative strategies in mental healthcare for cardiovascular disease in general. ${ }^{30}$ In summary, there is little evidence to support current strategies for venous thromboembolism prophylaxis in mental healthcare settings. Further study to develop and evaluate the effectiveness of novel venous thromboembolism prevention and early detection strategies is therefore warranted.

\section{About the author}

Dr Rashmi Patel is an MRC Clinical Research Training Fellow at the Department of Psychosis Studies, King's College London, UK.

\section{References}

1 Department of Health. Report of the Independent Expert Working Group on the Prevention of Venous Thromboembolism in Hospitalised Patients. Deparmtnet of Health, 2007 (http://webarchive.nationalarchives.gov.uk/ 20130107105354/http://www.dh.gov.uk/en/Publicationsandstatistics/ Publications/PublicationsPolicyAndGuidance/DH_073944).

2 National Institute for Health and Care Excellence. Venous Thromboembolism: Reducing the Risk: Reducing the Risk of Venous Thromboembolism (Deep Vein Thrombosis and Pulmonary Embolism) in Patients Admitted to Hospital. CG92. National Institute for Health and Care Excellence, 2010
3 Van Zyl M, Wieczorek G, Reilly J. Awareness of venous thromboembolism in mental health services for older people. J Psychiatr Ment Health Nurs 2014; 21: 375-8.

4 Falck-Ytter Y, Francis CW, Johanson N, Curley C, Dahl OE, Schulman S, et al. Prevention of VTE in orthopedic surgery patients: antithrombotic therapy and prevention of thrombosis, 9th ed: American College of Chest Physicians evidence-based clinical practice guidelines. Chest 2012; 141: e278S-325S.

5 Gould MK, Garcia D, Wren SM, Karanicolas PJ, Arcelus JI, Heit J, et al. Prevention of VTE in nonorthopedic surgical patients: antithrombotic therapy and prevention of thrombosis, 9th ed: American College of Chest Physicians evidence-based clinical practice guidelines. Chest 2012; 141: e227S-77S

6 Kahn SR, Lim W, Dunn AS, Cushman M, Dentali F, Akl Ea, et al. Prevention of VTE in nonsurgical patients: antithrombotic therapy and prevention of thrombosis, 9th ed: American College of Chest Physicians evidence-based clinical practice guidelines. Chest 2012; 141: e195S$226 \mathrm{~S}$

7 Lederle FA, Zylla D, Macdonald R, Wilt TJ. Venous thromboembolism prophylaxis in hospitalized medical patients and those with stroke: a background review for an American College of Physicians Clinical Practice Guideline. Ann Intern Med 2011; 155: 602-15.

8 Delluc A, Montavon S, Canceil O, Carpentier M, Nowak E, Mercier B, et al. Incidence of venous thromboembolism in psychiatric units. Thromb Res 2012; 130: e283-8.

9 Van Zyl M, Wieczorek G, Reilly J. Venous thromboembolism incidence in mental health services for older people: survey of in-patient units. Psychiatrist 2013; 37: 283-5.

10 White RH, Zhou H, Romano PS. Incidence of symptomatic venous thromboembolism after different elective or urgent surgical procedures. Thromb Haemost 2003; 90: 446-55.

11 Tsai AW, Cushman M, Rosamond WD, Heckbert SR, Polak JF, Folsom AR. Cardiovascular risk factors and venous thromboembolism incidence: the longitudinal investigation of thromboembolism etiology. Arch Intern Med 2002; 162: 1182-9.

12 Hägg S, Jönsson AK, Spigset O. Risk of venous thromboembolism due to antipsychotic drug therapy. Expert Opin Drug Saf 2009; 8: 537-47.

13 Masopust J, Mal R, Vali M. Risk of venous thromboembolism during treatment with antipsychotic agents. Psychiatry Clin Neurosci 2012; 66: 541-52.

14 De Hert M, Einfinger G, Scherpenberg E, Wampers M, Peuskens J. The prevention of deep venous thrombosis in physically restrained patients with schizophrenia. Int J Clin Pract 2010; 64: 1109-15.

15 Tsuda N, Sako A, Okamoto S, Adachi H, Hayakawa T, Makino K, et al. A significant association between physical restraint and the development of venous thromboembolism in psychiatric patients. Int J Cardiol 2012; 157: 442-3.

16 Ishida T, Katagiri T, Uchida H, Takeuchi H, Sakurai H, Watanabe K, et al. Incidence of deep vein thrombosis in restrained psychiatric patients. Psychosomatics 2014; 55: 69-75.

17 Greig MFG, Rochow SB, Crilly M, Mangoni A. Routine pharmacological venous thromboembolism prophylaxis in frail older hospitalised patients: where is the evidence? Age Ageing 2013; 42: 428-34.

18 Kopcke D, Harryman O, Benbow EW, Hay C, Chalmers N. Mortality from pulmonary embolism is decreasing in hospital patients. $J R$ Soc Med 2011; 104: 327-31.

19 Vaitkus PT, Leizorovicz A, Cohen AT, Turpie AGG, Olsson C-G, Goldhaber SZ, et al. Mortality rates and risk factors for asymptomatic deep vein thrombosis in medical patients. Thromb Haemost 2005; 93 : 76-9.

20 Smith-Bindman R, Miglioretti DL, Johnson E, Lee $C$, Feigelson HS, Flynn $M$, et al. Use of diagnostic imaging studies and associated radiation exposure for patients enrolled in large integrated health care systems, 1996-2010. JAMA 2012; 307: 2400-9.

21 Wiener RS, Schwartz LM, Woloshin S. When a test is too good: how CT pulmonary angiograms find pulmonary emboli that do not need to be found. BMJ 2013; 347.

\section{Bulletin}


22 Donato AA, Khoche S, Santora J, Wagner B. Clinical outcomes in patients with isolated subsegmental pulmonary emboli diagnosed by multidetector CT pulmonary angiography. Thromb Res 2010; 126: e266-70.

23 Mal R, Masopust J, Hosák L, Konupcíková K. Assessment of risk of venous thromboembolism and its possible prevention in psychiatric patients. Psychiatry Clin Neurosci 2008; 62: 3-8.

24 Wang TY, Honeycutt EF, Tapson VF, Moll S, Granger CB, Ohman EM. Incidence of thrombocytopenia among patients receiving heparin venous thromboembolism prophylaxis. Am J Med 2012; 125: 1214-21.

25 Health and Social Care Information Centre. Hospital Episode Statistics, Admitted Patient Care, England - 2012-13: Main Specialties. Health and Social Care Information Centre, 2013. (http://www.hscic.gov.uk/ catalogue/PUB12566/hosp-epis-stat-admi-main-spec-2012-13tab.xIsx).

26 Hogg K, Kimpton M, Carrier M, Coyle D, Forgie M, Wells P. Estimating quality of life in acute venous thrombosis. JAMA Intern Med 2013; 173: 1067-72
27 NHS England. National Venous Thromboembolism (VTE) Prevention Programme helping to save lives. NHS England, 2013. (http:// www.england.nhs.uk/2013/06/27/vte-prog/).

28 National Institute for Health and Care Excellence. Quality Standards Programme NICE Cost Impact and Commissioning Assessment: Quality Standard for Venous Thromboembolism (VTE) Prevention. National Institute for Health and Care Excellence, 2010 (http:// www.nice.org.uk/guidance/qs3/resources/qs3-vte-prevention-costimpact-and-commissioning-assessment).

29 Chang C-K, Hayes RD, Perera G, Broadbent MTM, Fernandes AC Lee WE, et al. Life expectancy at birth for people with serious mental illness and other major disorders from a secondary mental health care case register in London. PLoS One 2011; 6: e19590.

30 Bailey S, Gerada C, Lester H, Shiers D. The cardiovascular health of young people with severe mental illness: addressing an epidemic within an epidemic. Psychiatrist 2012; 36: 375-8. 